\title{
Chemiplasmonics for high-throughput biosensors
}

This article was published in the following Dove Press journal: International Journal of Nanomedicine

\author{
Achyut J Raghavendra ${ }^{1, *}$ \\ Jingyi Zhu',* \\ Wren Gregory' \\ Fengjiao Case' \\ Pradyumna Mulpur ${ }^{2}$ \\ Shahzad Khan ${ }^{3}$ \\ Anurag Srivastava ${ }^{3}$ \\ Ramakrishna Podila'
}

'Laboratory of Nano-biophysics, Clemson University, Clemson, SC 29634, USA; ${ }^{2}$ Clemson Nanomaterials Institute, Clemson University, Anderson, SC 29625, USA; ${ }^{3} \mathrm{ABV}$ Indian Institute of Information Technology and Management, Gwalior, MP, India

*These authors contributed equally to this work
Correspondence: Ramakrishna Podila Clemson Nanomaterials Institute, Clemson University, 8I Technology Dr, Suite S 200, Clemson, SC 29634, USA Email rpodila@g.clemson.edu
Background: The sensitivity of ELISA for biomarker detection can be significantly increased by integrating fluorescence with plasmonics. In surface-plasmon-coupled emission, the fluorophore emission is generally enhanced through the so-called physical mechanism due to an increase in the local electric field. Despite its fairly high enhancement factors, the use of surface-plasmoncoupled emission for high-throughput and point-of-care applications is still hampered due to the need for expensive focusing optics and spectrometers.

Methods: Here, we describe a new chemiplasmonic-sensing paradigm for enhanced emission through the molecular interactions between aromatic dyes and $\mathrm{C}_{60}$ films on $\mathrm{Ag}$ substrates.

Results: A 20-fold enhancement in the emission from rhodamine B-labeled biomolecules can be readily elicited without quenching its red color emission. As a proof of concept, we demonstrate two model bioassays using: 1) the RhB-streptavidin and biotin complexes in which the dye was excited using an inexpensive laser pointer and the ensuing enhanced emission was recorded by a smartphone camera without the need for focusing optics and 2) high-throughput 96-well plate assay for a model antigen (rabbit immunoglobulin) that showed detection sensitivity as low as $6.6 \mathrm{pM}$. Conclusion: Our results show clear evidence that chemiplasmonic sensors can be extended to detect biomarkers in a point-of-care setting through a smartphone in simple normal incidence geometry without the need for focusing optics. Furthermore, chemiplasmonic sensors also facilitate high-throughput screening of biomarkers in the conventional 96-well plate format with 10-20 times higher sensitivity.

Keywords: biosensor, surface plasmons, nanosilver, fluorescence, fullerenes

\section{Introduction}

The realization of highly sensitive, portable, rapid, and inexpensive biosensors is necessary to improve patient care through real-time disease monitoring and diagnosis at the point of care (POC). ${ }^{1-4}$ The recent advances in genomics and proteomics have led to the identification of many potential biomarkers for complex diseases. However, the widespread use of these biomarkers has been limited due to the lack of efficient POC biosensors. ${ }^{1}$ For example, the WHO estimated that only $67 \%$ of tuberculosis cases were diagnosed in time in 2014, which is a mere $4 \%$ improvement over the global detection rate of $63 \%$ in $2009 .{ }^{1,5}$ The WHO and the US National Institutes of Health clearly emphasize that fundamental breakthroughs in POC biosensing are imperative for realizing nearly $100 \%$ early disease detection and treatment. ${ }^{6}$

In the last two decades, surface plasmons from metallic nanoparticles (NPs) have been widely used to improve the detection limits for many biomarkers through fluorescence enhancements. ${ }^{7-15}$ Metallic NPs are known to alter fluorescence emission of dyes by influencing the radiative of dye molecules within their vicinity. ${ }^{16}$ The radiative decay rate $(\gamma)$ of a dye molecule, from an excited state $\mid k>$ of energy $E_{k}$ to a lower state $\mid n>$ of energy $E_{n}$, through the emission of a photon of energy $\hbar \omega$, may be understood in terms of the Fermi's golden rule given by 


$$
\gamma=\frac{2 \pi}{\hbar} \sum_{n \neq k}|<n| \vec{p} \cdot \vec{E}|k>|^{2} \rho(r, \omega)
$$

In Equation 1, $\hbar$ is reduced Planck's constant, $\vec{p}$ is the dipole moment of the fluorescent molecule, $\vec{E}$ is the total electric field. The term $\rho(r, \omega)$ in Equation 1 is the photonic mode density (PMD), which is defined as the ratio of photon flow per unit area per second (ie, power flow per photon) to the velocity of photons. Similar to the electronic density of states in solid-state physics, PMD provides the density of photonic states and describes the strength of interaction between a fluorescent molecule and its photonic environment. The PMD for a dipole $\vec{p}$ aligned along the direction $\hat{e}_{d}$ can be expressed in terms of the field term $\left(\hat{e}_{d} \cdot \vec{G}\left(r, r^{\prime}, \omega\right) \cdot \hat{e}_{d}\right)$ containing the dyadic Green's function $\left(\vec{G}\left(r, r^{\prime}, \omega\right)\right)$ of the system in which the dye is embedded. ${ }^{17}$ The presence of metallic NPs is known to change the decay rate and enhance fluorescence emission by increasing PMD. ${ }^{18,19}$ Previously, we and others have demonstrated surface-plasmon-coupled emission sensors that enhance the fluorescence of dyes by increasing PMD in the presence of $\mathrm{Ag} / \mathrm{Au} \mathrm{NPs}$ and thin films. ${ }^{7-15}$ Here, we posit that it is also plausible to enhance fluorescence through chemical interactions between the fluorescent molecule and the photonic environment that change the matrix element (ME) $<n|\vec{p} \cdot \vec{E}| k>$ in Equation 1 in addition to increasing PMD.

In this article, we describe a stacked $\mathrm{Ag} / \mathrm{C}_{60}$ POC platform to increase fluorescence emission through a new chemiplasmonic mechanism that simultaneously increases both ME and PMD. We achieved emission enhancement factors as high as 20 without the need for any lenses or other focusing optics, which are used extensively in surface-plasmon-coupled emission-based sensors. Unlike the physical mechanism that enhances only PMD, the chemiplasmonic mechanism also influences $\mathrm{ME}$ by selectively restricting the nonradiative rotational modes of dye molecules through $\pi-\pi$ interactions with the $\mathrm{C}_{60}$ film. We show that the emission from the dye molecules on a chemiplasmonic platform can be excited via normally incident unpolarized light from an inexpensive laser pointer and efficiently captured using a smartphone camera. We further demonstrate that the spectra captured through the smartphone can be analyzed via an inbuilt app for obtaining quantified data for a specific biomarker. We validate a POC model assay using biotin-streptavidin (SA) complex on vertically stacked $\mathrm{Ag} / \mathrm{C}_{60}$ layers. Lastly, we demonstrate that chemiplasmonics when integrated into a high-throughput 96-well plate model assay for a model antigen (rabbit immunoglobulin or IgG), a 10-fold enhancement in emission can be elicited, resulting in a detection limit as low as $6.6 \mathrm{pM}$.

\section{Methods}

RhB (>95\%) was purchased from Sigma-Aldrich, St. Louis, MO, USA. B-BSA was obtained from Thermo Scientific, Waltham, MA, USA, while SA-RhB was from Bio-Synthesis, Inc (Lewisville, TX, USA). Glass coverslips (Sigma-Aldrich) were used as the base substrate. As described in the study by Mulpur et al, ${ }^{26}$ coverslips were coated with either $25 \mathrm{~nm}$ of Ag using a home built thermal evaporator or 10-35 $\mathrm{nm} \mathrm{C}_{60}$ (99.9\%; SES Research, Houston, TX, USA). For studying the emission characteristics, $1 \mathrm{mM}$ solution of $\mathrm{RhB}$ was spin coated onto the substrates at 3,000 rpm (KW-4A; Chemat Technology, Northridge, CA, USA). For physical adsorption isotherm studies, glass slides with $\mathrm{Ag}$ and different thickness of $\mathrm{C}_{60}$ were incubated in different concentrations of RhB in de-ionized water $(0.1 \mathrm{M}$, $0.01 \mathrm{M}, 1 \mathrm{mM}, 0.1 \mathrm{mM}, 0.01 \mathrm{mM}$ ) for 24 hours after which they were taken out and washed in de-ionized water thrice and dried in an air stream. For studies with b-BSA, substrates were prepared as detailed in the study by Ray et al. ${ }^{27}$ Briefly, $\sim 2.5 \mu \mathrm{M}$ b-BSA solution was made in $10 \mathrm{mM}$ PBS (Sigma-Aldrich) solution. Glass, glass-Ag, $\mathrm{C}_{60}$, and glass$\mathrm{Ag}-\mathrm{C}_{60}$ substrates were then incubated in this solution for 24 hours at $51{ }^{\circ} \mathrm{C}$. The substrates were taken out and washed in PBS solution at least three times to remove any unattached b-BSA. The samples were then incubated in $2 \mathrm{nM}$ solution of SA-RhB in PBS solution at $51^{\circ} \mathrm{C}$ for 2 hours. They were then taken out and washed several times in PBS solution and dried in air. The photoluminescence spectra were collected by exciting the samples using an $\mathrm{Ar}^{+} 514.5$ $\mathrm{nm}$ excitation (laser power $\sim 0.1 \mathrm{~mW}$ ) coupled to a Renishaw inVia spectrometer. All the spectra were normalized by the incident laser intensity. The spectra were fit using IGOR Pro by deconvoluting each spectrum into multiple Lorentzian functions with similar widths. A fit was deemed good for $R^{2}$ (coefficient of determination) values $>0.95$. All the enhancement values for different substrates were obtained by dividing the peak enhancement intensity by RhB peak emission intensity on glass slide (Figure 1A). Perkin Elmer Lambda 950 was used to collect UV-Vis absorption spectra of the samples. Fluorescence microscopy images were taken using Zeis AXIO Imager A1 coupled to X-cite series $120 \mathrm{~W}$ Xe lamp at $20 \times$ magnification. The samples were excited using $532 \mathrm{~nm}$ light from $120 \mathrm{~W}$ Xe lamp, and the emission was collected through $532 \mathrm{~nm}$ high-pass filter. For smartphone camera sensing, the samples were excited using 
a $10 \mathrm{~mW}$ green laser pointer (HK-E00187; Laserpointer Pro, North Brunswick, NJ, USA). A long-pass filter with a cut-on wavelength $\sim 550 \mathrm{~nm}$ (FEL0550; Thorlabs, Newton, NJ, USA) was taped on to the smartphone (iPhone $5 \mathrm{~s}$ ) for capturing the emitted light through the camera. The obtained pictures of emitted light were analyzed using ImageJ software to obtain the RGB content. The DFT calculations were performed using Gaussian 09 package. We used a hybrid Becke-3-Lee-Yang-Parr (B3LYP) exchange correlation with Lan12DZ basis set (a pseudopotential approach) for the ground state calculations.

The 96-well plate was noncovalently coated with rabbit IgG. A coating solution of $\operatorname{IgG}(10-30 \mu \mathrm{g} / \mathrm{mL}$ of IgG dissolved in Na-phosphate buffer, $50 \mathrm{mM}, \mathrm{pH}$ 7.4) was added to each well $(25 \mu \mathrm{L} /$ well $)$, and slides were incubated for $2-4$ hours at room temperature in a humid chamber. Slides were then rinsed with de-ionized water, washing solution $(0.05 \%$ Tween 20 in water), and de-ionized water. Blocking was performed by adding blocking solution (1\% BSA, $1 \%$ sucrose, $0.05 \% \mathrm{NaN} 3$, and $0.05 \%$ Tween- 20 in $50 \mathrm{mM}$ Tris$\mathrm{HCl}$ buffer, $\mathrm{pH} 7.4,35 \mu \mathrm{L}$ per well) and incubating at room temperature for $2-4$ hours (or overnight at $4^{\circ} \mathrm{C}$ ) in a humid chamber. The plate was then rinsed with de-ionized water, washing solution ( $0.05 \%$ Tween- 20 in water), and de-ionized water, covered with Na-phosphate buffer $(50 \mathrm{mM}, \mathrm{pH} 7.4)$ or blocking solution, and stored at $4^{\circ} \mathrm{C}$ until use. Dye-labeled conjugate dye antirabbit IgG (diluted to $10 \mu \mathrm{g} / \mathrm{mL}$ with Na-phosphate buffer, $50 \mathrm{mM}$, pH 7.4) was added to the wells (coated with rabbit $\operatorname{IgG}$ ) and incubated at room temperature in a humid chamber for 1-2 hours. The plate was then rinsed with de-ionized water, washing solution $(0.05 \%$ Tween 20 in water), and de-ionized water, coated with blocking buffer, and stored at $4^{\circ} \mathrm{C}$ before measurement. A scheme of the model immunoassay is presented in Figure 9A.

\section{Results and discussion}

As shown in Figure 1A, the emission of rhodamine $\mathrm{B}$ $(\mathrm{RhB})$ on $\mathrm{Ag} / \mathrm{C}_{60}$ layers evolves with the thickness of $\mathrm{C}_{60}$. Indeed, the emission intensity was found to be maximum when the $\mathrm{C}_{60}$ thickness is $\sim 10-20 \mathrm{~nm}$ (Figure 1B). Such enhancement can be understood in terms of the well-known metal-enhanced fluorescence (MEF) model, which describes
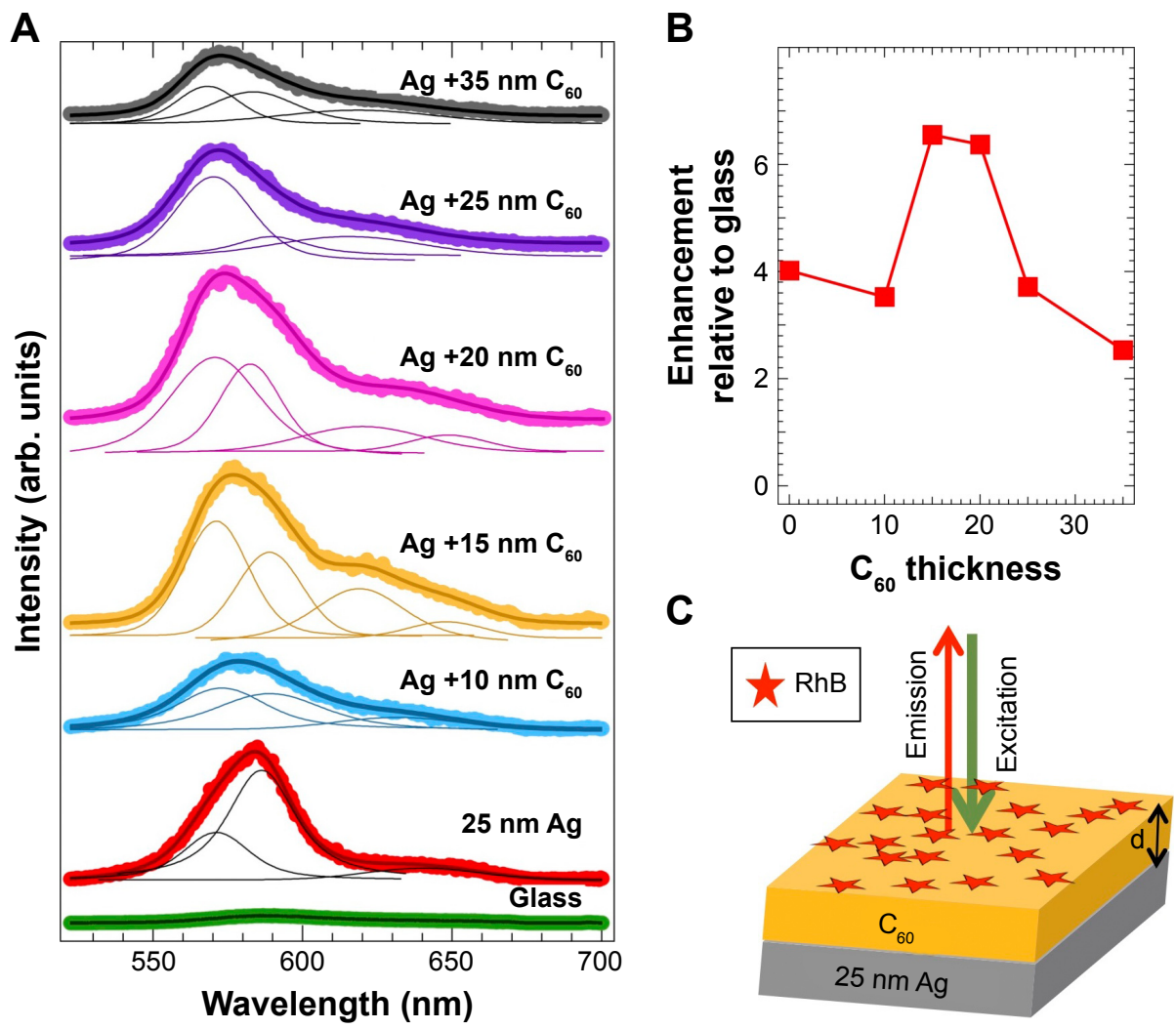

Figure I (A) The emission spectra of rhodamine B (RhB) adsorbed on bare glass and $25 \mathrm{~nm}$ Ag-coated glass with increasing thicknesses of $C_{60}(0-35 \mathrm{~nm})$. The solid traces represent fits to the spectra, from which the deconvoluted peaks for each spectrum were obtained. The area under the spectra was used to calculate the enhancement factors (relative to glass) as a function of $\mathrm{C}_{60}$ thickness shown in (B). (C) Schematic of the chemiplasmonic substrate used in this study for obtaining the emission spectra in (A). 

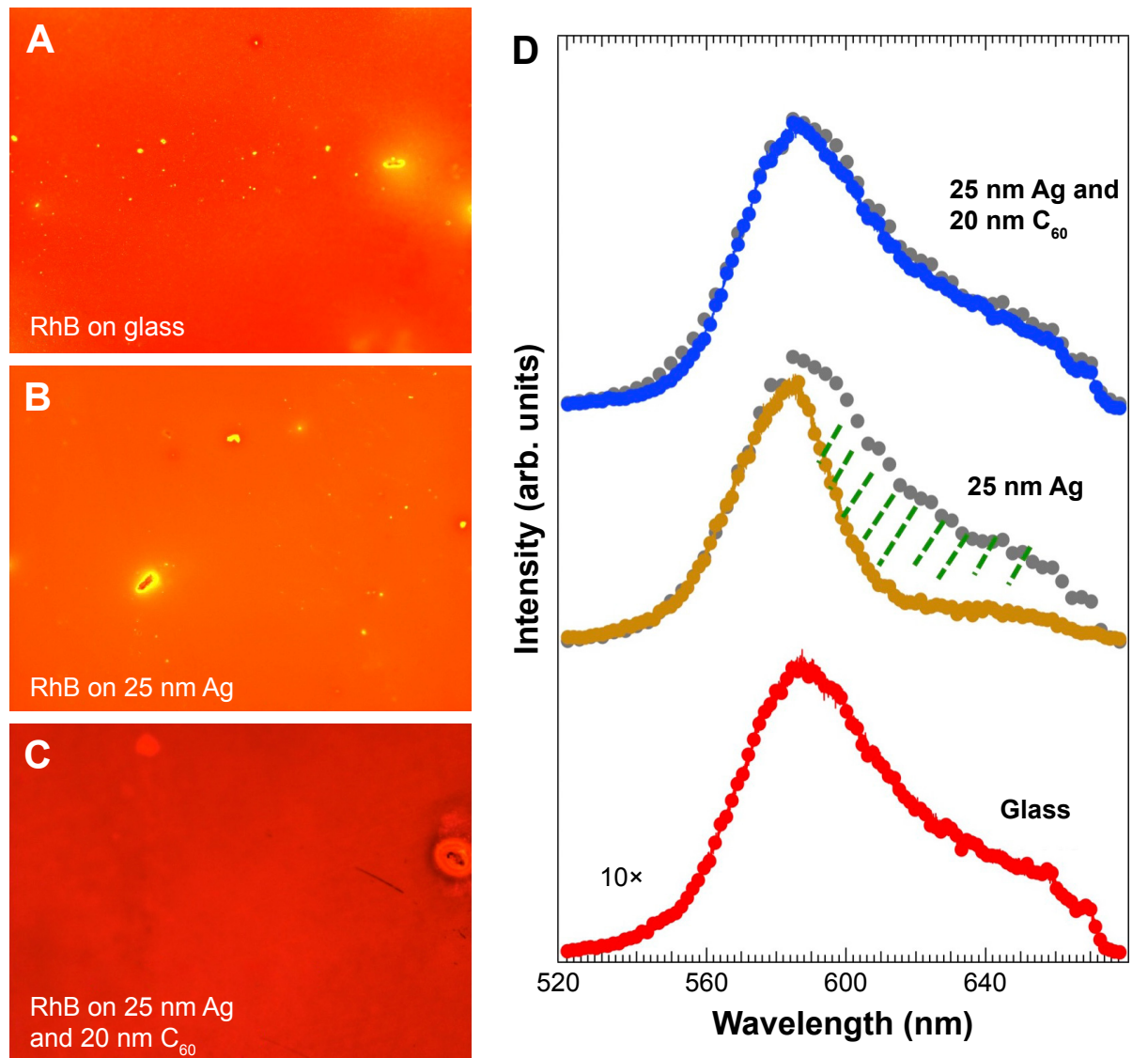

Figure 2 Fluorescence microscope images of rhodamine B (RhB)-coated glass (A), glass $+25 \mathrm{~nm} \mathrm{Ag}(\mathbf{B})$, and glass $+25 \mathrm{~nm} \mathrm{Ag}+20 \mathrm{~nm} \mathrm{C}$ (C). The bright spots arise from accumulated RhB. The figures are I00×100 microns. The magnification is I0X. (D) The corresponding RhB emission spectra from different substrates (glass, $25 \mathrm{~nm}$ Ag, $20 \mathrm{~nm}$ $\mathrm{C}_{60}$, and $25 \mathrm{~nm} \mathrm{Ag}+20 \mathrm{~nm} \mathrm{C}_{60}$ ) clearly show that the $\mathrm{Ag}$ layer quenches the red emission from RhB (shown by green hatched area in $25 \mathrm{~nm} \mathrm{Ag}$ spectrum). The gray colored circles in the top two spectra show the magnified $(\times 10)$ RhB spectrum on the glass for comparison.

changes in emission or quenching of a fluorophore near the surface of a thin metal film. ${ }^{15,20}$ In the MEF framework, excited fluorophores are modeled as oscillating dipoles and their interaction with a metal surface is described through two concomitant mechanisms. The first of these is the metalinduced damping of the dipole oscillators that quenches the emission. ${ }^{15,21}$ The strength of such damping decreases with increasing distance $(d)$ from the metal surface (Figure 1C) and follows a $1 / d^{3}$ dependence. ${ }^{15,21}$ The second mechanism is the plasmon-assisted increase in the electric field, which enhances emission and decreases with a $1 / d^{6}$ dependence. ${ }^{15,21}$ Collectively, these mechanisms result in a distance-dependent enhancement in the fluorescence emission of the dye, as evidenced in Figure 1B. A maximum of six-fold enhancement in the $\mathrm{RhB}$ emission on $\mathrm{Ag}(25 \mathrm{~nm}) / \mathrm{C}_{60}$ was recorded when the $\mathrm{C}_{60}$ film thickness was $\sim 10-20 \mathrm{~nm}$ (Figure 1B).

Interestingly, the line shape and width of the fluorescence spectrum are significantly influenced by the $\mathrm{C}_{60}$ film thickness. Although many previous studies explained the emission enhancements through the MEF model, changes in the spectral features, such as in Figure 1A, were partly ignored. ${ }^{8,9,12,13,22,23}$ While the emission spectrum of RhB on $25 \mathrm{~nm} \mathrm{Ag}$ required a two-peak fit, at least three to four peaks were necessary to fit the emission spectrum of $\mathrm{RhB}$ on $\mathrm{Ag} /$ $\mathrm{C}_{60}$ layers. This important fact is also reflected in Figure $2 \mathrm{~A}-\mathrm{C}$, which show fluorescence microscope images of $\mathrm{RhB}$ coated on bare glass, $25 \mathrm{~nm} \mathrm{Ag}$, and $25 \mathrm{~nm} \mathrm{Ag} / 20 \mathrm{~nm} \mathrm{C}$. Clearly, the emission of $\mathrm{RhB}$ present on $\mathrm{Ag}$ appears orange unlike the emission of $\mathrm{RhB}$ on either glass or $\mathrm{Ag} / 20 \mathrm{~nm} \mathrm{C}_{60}$ substrates that display a distinct red color. A closer examination of the emission spectra (Figure 2D) revealed that the red emission (above $600 \mathrm{~nm}$ ), which is otherwise present in $\mathrm{RhB}$ coated on glass, is 1) completely quenched when $\mathrm{RhB}$ is coated on a $25 \mathrm{~nm}$ thick $\mathrm{Ag}$ substrate and 2) remains unaffected when $\mathrm{RhB}$ is coated on $20 \mathrm{~nm}$ bare $\mathrm{C}_{60}$ film (without $\mathrm{Ag}$ ). These observations suggest that in the former case (ie, case 1) the Ag films strongly quenched RhB emission in the red region. Similar to bare Ag films, the emission spectrum 

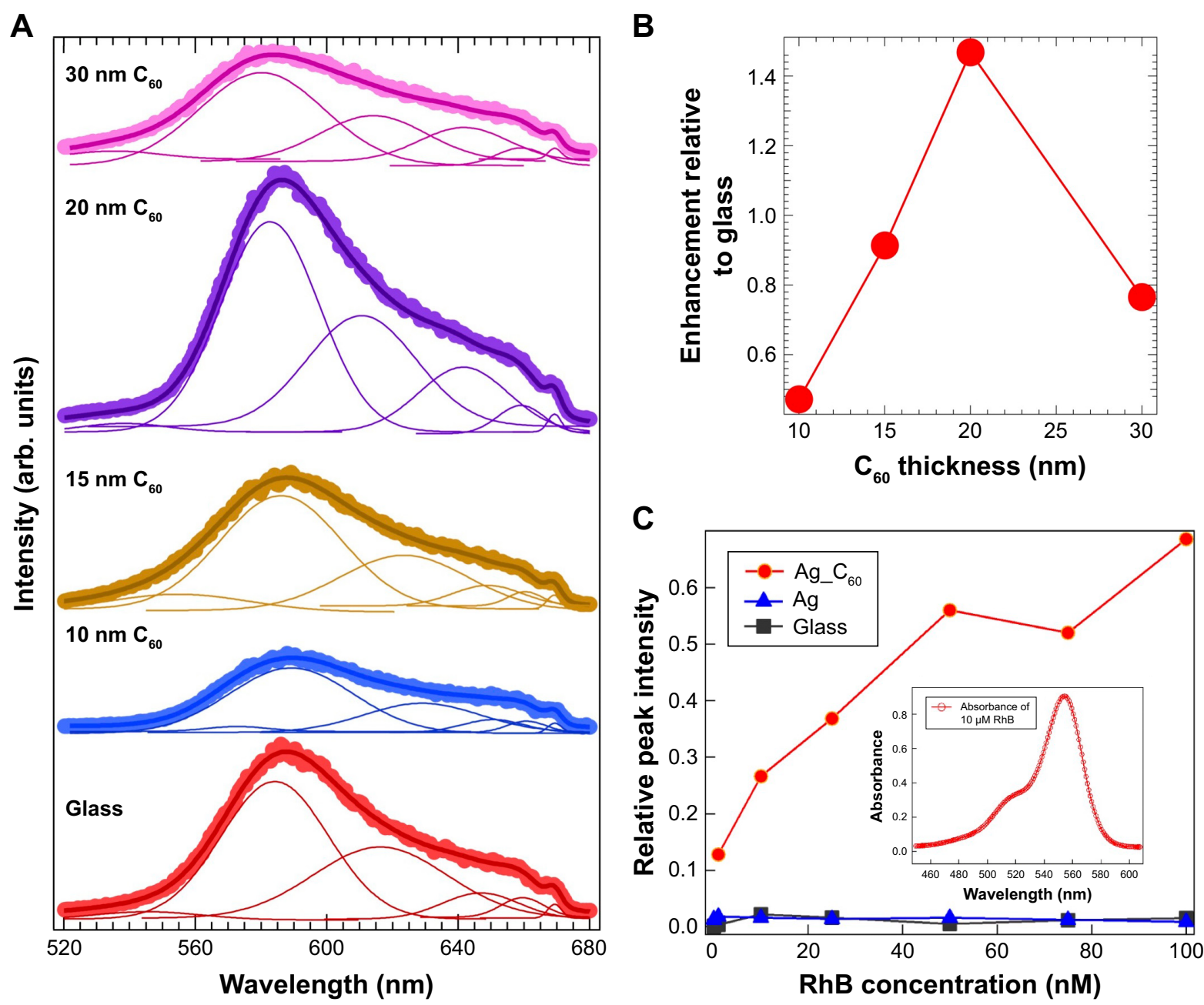

Figure 3 (A) The emission spectra of rhodamine $B(\mathrm{RhB})$ adsorbed on a bare glass substrate with increasing thickness of $\mathrm{C}_{60}(0-30 \mathrm{~nm})$. The solid lines show the fits to the spectra along with the deconvoluted peaks under each spectrum. (B) When the $C_{60}$ film thickness reached 20 nm, an emission enhancement of $\sim 1.5$ was observed due to the $\pi-\pi$ interactions between the RhB molecules and $C_{60}$ (C) The adsorption isotherms for RhB on $C_{60}$ show an increasing trend unlike $25 \mathrm{~nm} A g$ or bare glass substrates, suggesting the presence of strong $\pi$-orbital interactions between $\mathrm{C}_{60}$ and $\mathrm{RhB}$.

was found to shift to higher wavelengths for conventional spacer layers such as $\mathrm{SiO}_{2}$ (Figure $\mathrm{S} 1$ ). We hypothesize that $\mathrm{C}_{60}$ is able to retain the original features of $\mathrm{RhB}$ emission spectrum through $\pi-\pi$ stacking, which is discussed later in Figure 3C.
Remarkably, RhB on bare $\mathrm{C}_{60}$ (without Ag) also exhibited a non-negligible thickness-dependent enhancement (Figure 3A and B). This enhancement, unlike the physical mechanism in case of $\mathrm{Ag}-\mathrm{RhB}$, arises due to the chemical interactions between $\mathrm{C}_{60}$ and $\mathrm{RhB}$ molecules. We surmise
A

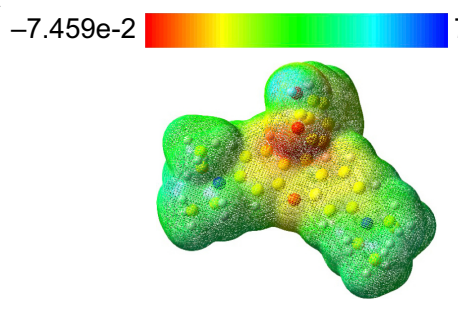

B

$7.459 e-2$

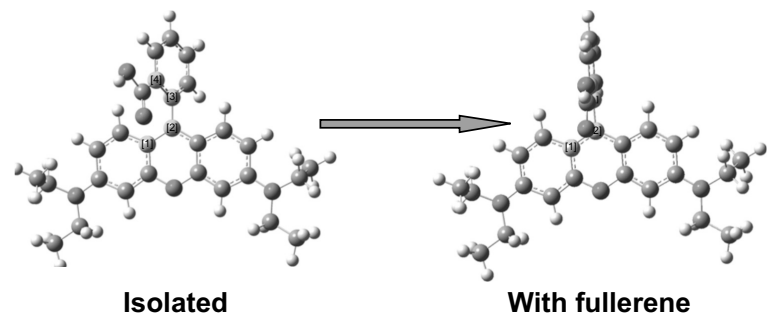

Figure 4 (A) The molecular electrostatic potential for rhodamine B (RhB) shows that its carboxylic group is electron rich (yellow color). (B) The interaction of RhB with $\mathrm{C}_{60}$ results in the rotation of its carboxylic group in the benzoic acid above the xanthene ring by $32^{\circ}$. 

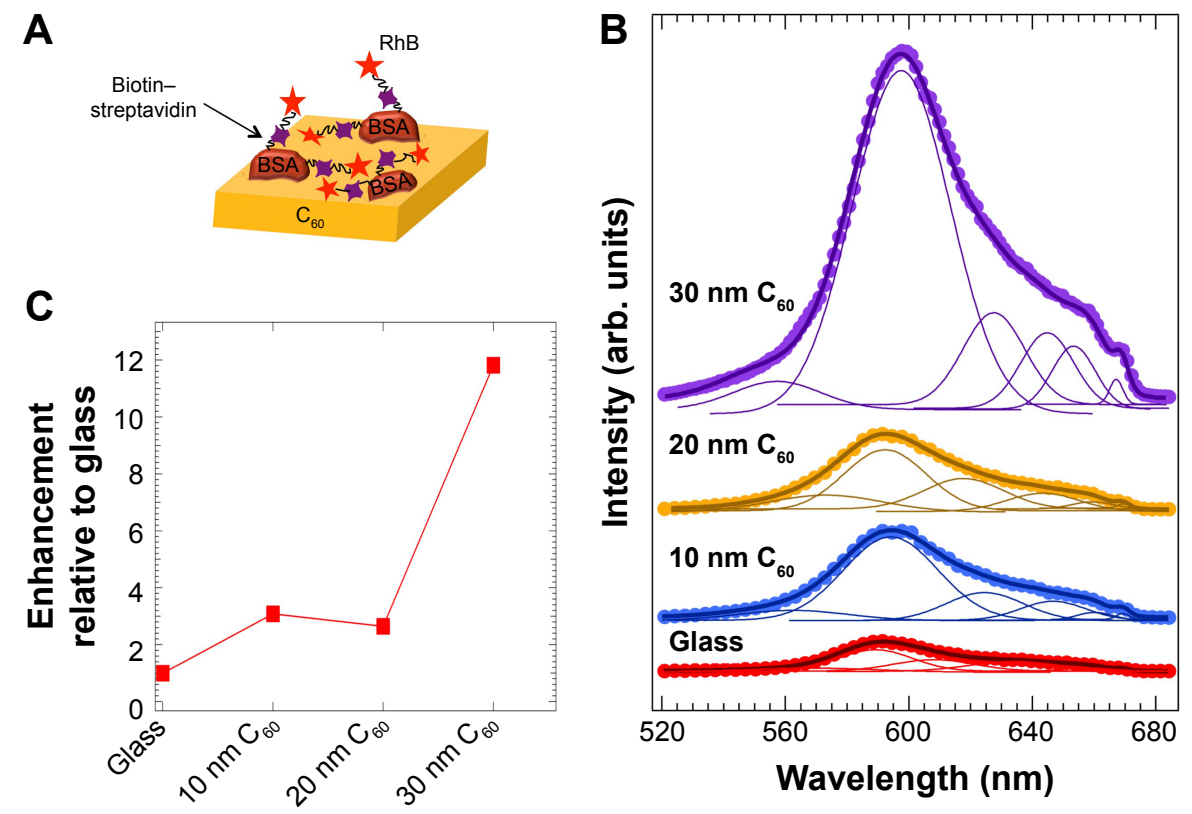

Figure 5 (A) A schematic showing the model biotin-streptavidin assay on pure $C_{60}$ layers of varying thickness. The biotin-streptavidin is flexible to allow the direct interaction between rhodamine $B(R h B)$ attached to streptavidin with the underlying $C_{60}$ layers. (B) The emission spectrum of the biotin-streptavidin-RhB complex on $C_{60}$ layers with varying thickness displays a clear enhancement (shown in (C)) due to the chemical interactions between $\mathrm{C}_{60}$ and $\mathrm{RhB}$. The solid lines in (B) show the fits to the spectra along with the deconvoluted peaks under each spectrum.

that the $\pi$-orbitals in $\mathrm{C}_{60}$ and $\mathrm{RhB}$ can result in $\pi-\pi$ stacking $^{8}$ and thereby influence the $\mathrm{ME}$ in Equation 1 by restricting those rotational/vibrational modes of $\mathrm{RhB}$ molecules that would otherwise participate in the nonradiative decay of excited electrons. We obtained adsorption isotherms of $\mathrm{RhB}$ on $\mathrm{Ag} / \mathrm{C}_{60}$ substrates to confirm the presence of $\pi-\pi$ interactions (Figure 3C). Using the maximum peak intensity in the absorption spectrum of $\mathrm{RhB}$ (inset in Figure 3C) as an indicator for the amount of $\mathrm{RhB}$ adsorbed on the substrates, it is evident from Figure $3 \mathrm{C}$ that the adsorption of $\mathrm{RhB}$ on $\mathrm{Ag} / \mathrm{C}_{60}$ is stronger and follows a Freundlich-like behavior (indicative of multilayered adsorption) unlike $\mathrm{RhB}$ on glass or Ag. More importantly, as shown in Figure S2, the emission of $\mathrm{RhB}$ on $\mathrm{C}_{60}$ substrates was found to be predominantly $p$-polarized (unlike $\mathrm{RhB}$ on glass or $\mathrm{Ag}$ ), indicating that the $\mathrm{RhB}$ molecules assume a preferred orientation on $\mathrm{C}_{60}$ due to $\pi-\pi$ interactions.

To further examine the nature of these interactions, we investigated the molecular electrostatic potential of $\mathrm{RhB}$ (Figure 4A). We found that the dangling oxygen of carboxylic group (in benzoic acid present below the xanthene ring) has electron-rich regions and may serve as a donor. It should be noted that the highest-occupied and lowest unoccupied molecular orbital levels of $\mathrm{RhB}\left(/ \mathrm{C}_{60}\right)$ are located at $5.6 \mathrm{eV}(/ 6.2 \mathrm{eV})$ and $-3.5 \mathrm{eV}(/-4.1 \mathrm{eV})$ with respect to vacuum indicating that $\mathrm{C}_{60}$ is a suitable acceptor. ${ }^{24,25}$ Such charges transfer interactions and the mixing of $\mathrm{RhB}$ molecular orbitals with $\mathrm{C}_{60}$ electronic states could result in an enhanced emission through the so-called chemical mechanism due to an increase in ME in Equation 1. Our density functional theory (DFT) calculations (Figure 4B) showed that the benzoic acid group rotates by $32^{\circ}$ upon interaction with $\mathrm{C}_{60}$ and thereby restricts some of the rotational modes in RhB. Our DFT results further suggest that the observed chemical enhancement in $\mathrm{RhB}$ emission on bare $\mathrm{C}_{60}$ could possibly result from changes to $\mathrm{ME}$ (Equation 1) through both charge transfer between $\mathrm{RhB}$ and $\mathrm{C}_{60}$ and the restriction of rotational motion of the benzoic acid group. Figure S3 shows the temperature dependence of enhancement factor of $\mathrm{RhB}$ on $\mathrm{Ag}, 20 \mathrm{~nm}$ $\mathrm{C}_{60}$, and $\mathrm{Ag} / 20 \mathrm{~nm} \mathrm{C}_{60}$. As expected, the enhancement factor reduces with increasing temperature due to the increased molecular vibrations, which rapidly decrease the radiative lifetime. While Ag film showed a linear decrease in the enhancement factor with temperature, $\mathrm{C}_{60}$ films (both bare $\mathrm{C}_{60}$ and $\mathrm{Ag} / \mathrm{C}_{60}$ ) exhibited a stable enhancement factor up to $55^{\circ} \mathrm{C}$ followed by a sudden decrease above $55^{\circ} \mathrm{C}$. This suggests that at higher temperatures $\left(>55^{\circ} \mathrm{C}\right)$ the benzoic acid groups in $\mathrm{RhB}$ on $\mathrm{C}_{60}$ overcome the restriction imposed (Figure 4 ) by its binding with $\mathrm{C}_{60}$. The maximum observed chemical enhancement of $\mathrm{RhB}$ emission on bare $\mathrm{C}_{60}$ was $\sim 1.4$, which is consistent with the $\sim 1.5$ enhancement 
A

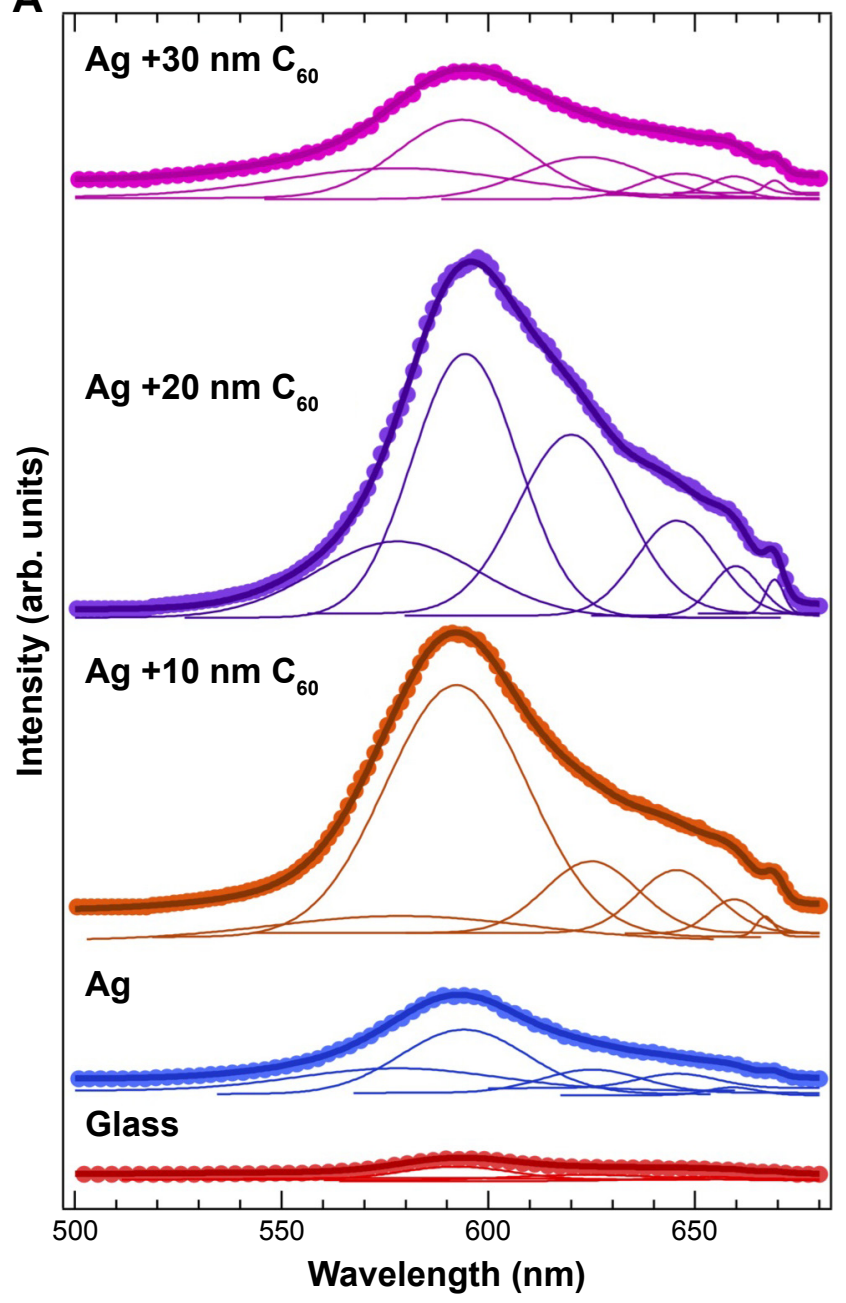

B

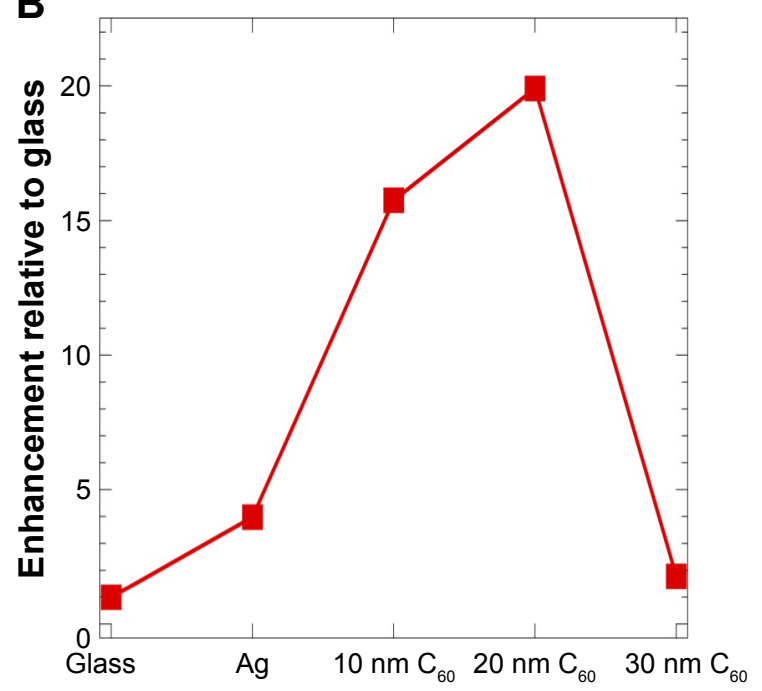

C

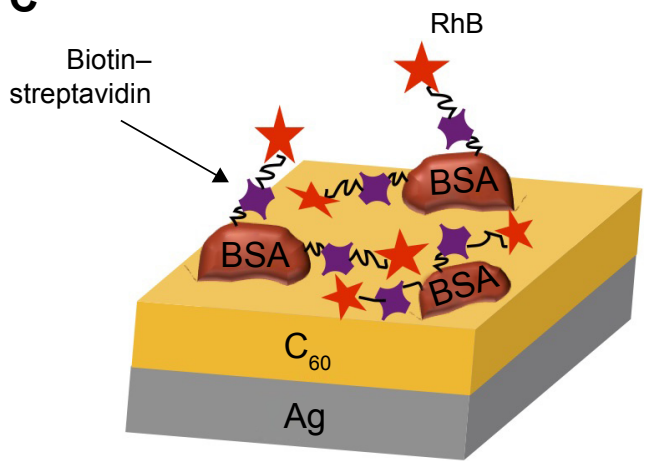

Figure 6 (A) The emission spectra of biotin-streptavidin-rhodamine B (RhB) adsorbed on the pure glass and $25 \mathrm{~nm}$ Ag-coated glass with different thicknesses of $\mathrm{C}_{60}$ $(0-30 \mathrm{~nm})$. The solid lines show the fits to the spectra along with the deconvoluted peaks under each spectrum. The area under the spectra was used to calculate the enhancement factors relative to glass shown in (B). (C) A schematic showing the model biotin-streptavidin assay on $\mathrm{Ag}+\mathrm{C}_{60}$ layers of varying thickness geometry that was used for obtaining the emission spectra in $(\mathbf{A})$.

in Figure 3B. These low enhancement factors are attributed to self-quenching, which possibly arises from multilayer adsorption of $\mathrm{RhB}$ molecules on $\mathrm{C}_{60}$, akin to the behavior of the adsorption isotherms in Figure 3C.

We alleviated the problems associated with $\mathrm{RhB}$ multilayer adsorption through the use of the biotin-SA complex. We first covered the bare $\mathrm{C}_{60}$ surface (without Ag) with biotinylated BSA (b-BSA) and then coated it with RhB-labeled SA (Figure 5A). Biotin and SA have strong nonspecific interactions that lead to binding of four biotin molecules to each SA. Such selective binding between biotin and SA mitigates multilayer adsorption of dye molecules when RhB-labeled SA attaches exclusively to b-BSA. As depicted in Figure 5B and $C$, the bare $\mathrm{C}_{60}$ films (without $\mathrm{Ag}$ ) showed a 12-fold enhancement in the emission upon lessening the aggregation-induced self-quenching of $\mathrm{RhB}$.
This enhancement is rather intriguing particularly because the $\mathrm{RhB}$ molecules are not directly present on the $\mathrm{C}_{60}$ surface unlike in Figure 3. It may be rationalized that the biotin-SA complex is sufficiently flexible due to its noncovalent nature and supports chemical interaction between $\mathrm{RhB}$ and $\mathrm{C}_{60}$ (Figure 5A).

We combined the chemical enhancement observed in $\mathrm{C}_{60}$-biotin-SA-RhB system with the physical mechanism by incorporating a $25 \mathrm{~nm}$ thick $\mathrm{Ag}$ layer (Figure 6A). This synergistic combination displayed much higher enhancement $\sim 20$-fold (Figure $6 \mathrm{~B}$ and $\mathrm{C}$ ) than either mechanism ( $\sim 6$-fold for physical and $\sim 12$-fold for chemical). We found that the maximum enhancement occurred $\sim 15-20 \mathrm{~nm}$ $\mathrm{C}_{60}$ thickness in Figure 6B similar to the enhancements observed in Figure 1B. Indeed, these enhancements are also apparent in the fluorescence microscope images 

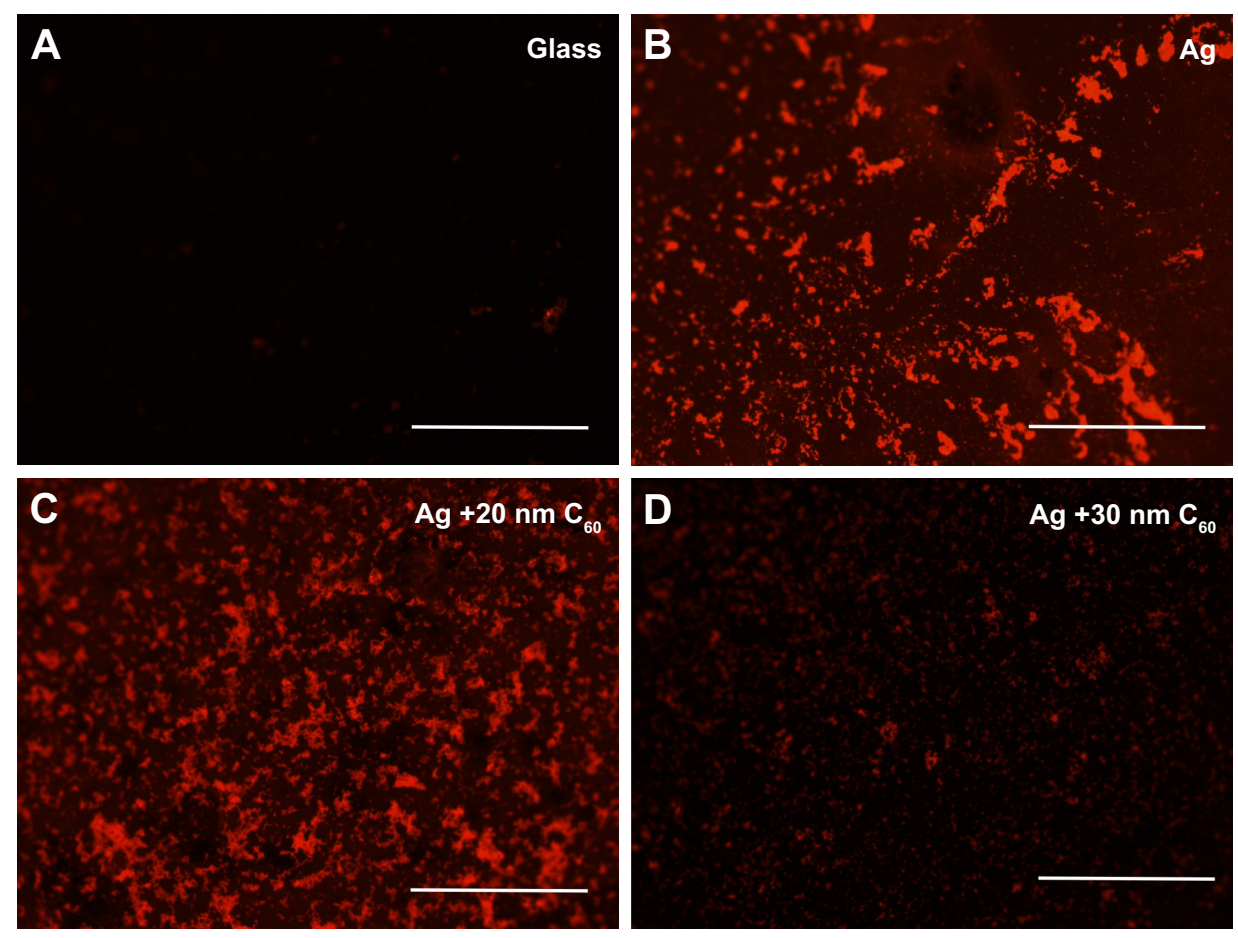

Figure 7 Fluorescence microscope images of biotin-streptavidin-rhodamine $B(R h B)$ on glass $(\mathbf{A})$, glass $+25 \mathrm{~nm} \mathrm{Ag}(\mathbf{B})$, glass $+25 \mathrm{~nm} \mathrm{Ag}+20 \mathrm{~nm} \mathrm{C}(\mathbf{C})$, and glass $+25 \mathrm{~nm}$ $\mathrm{Ag}+30 \mathrm{~nm} \mathrm{C}$ (D). All the scale bars are $50 \mu \mathrm{m}$; the magnification is $10 \times$.

of biotin-SA-RhB on different substrates shown in Figure 7A-D. While the RhB-labeled SA is not visible on the bare glass substrate, they can be clearly observed on $\mathrm{Ag}$ and $\mathrm{Ag} / 20 \mathrm{~nm} \mathrm{C}_{60}$ substrates. Not surprisingly, the presence of biotin-SA complex between $\mathrm{RhB}$ and bare Ag serves as a spacer material and thus slightly reduced (but did not eliminate) the effects of metal-induced quenching of red emission in RhB (discussed earlier in

A
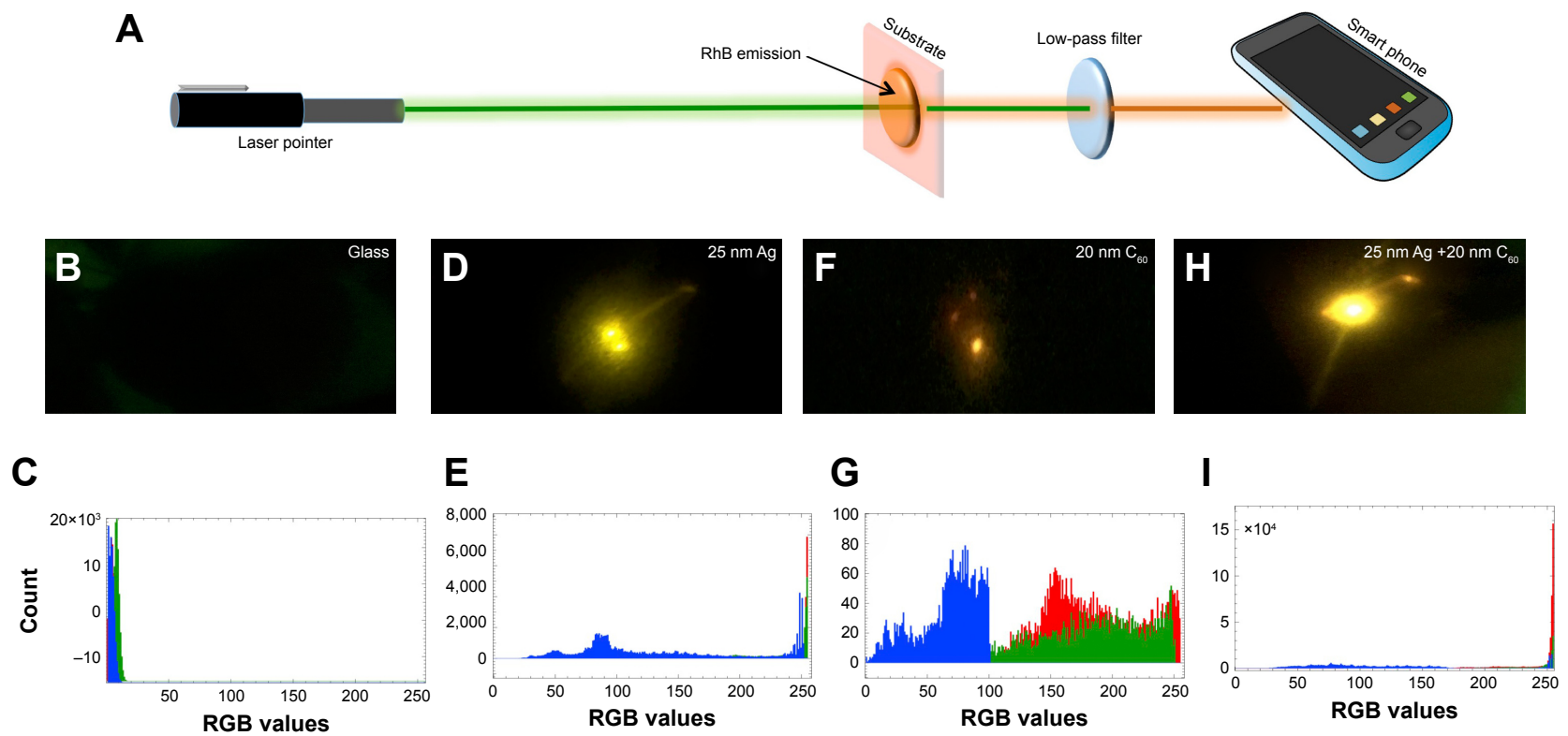

Figure 8 (A) A schematic showing the setup for smartphone-based sensing. A laser pointer emits green light to excite rhodamine B (RhB) coated on different substrates (shown in pink). The excited RhB molecules emit orange-red light, which could be captured by the smartphone through a low-pass filter. (B-I) The photographs of RhB emission obtained using a smartphone. The photographs were analyzed using Image software for obtaining the red, green, blue values. It should be noted that RGB values are integer values ranging between 0 and 255 and allow for the identification of $\sim 16.7$ million colors. 

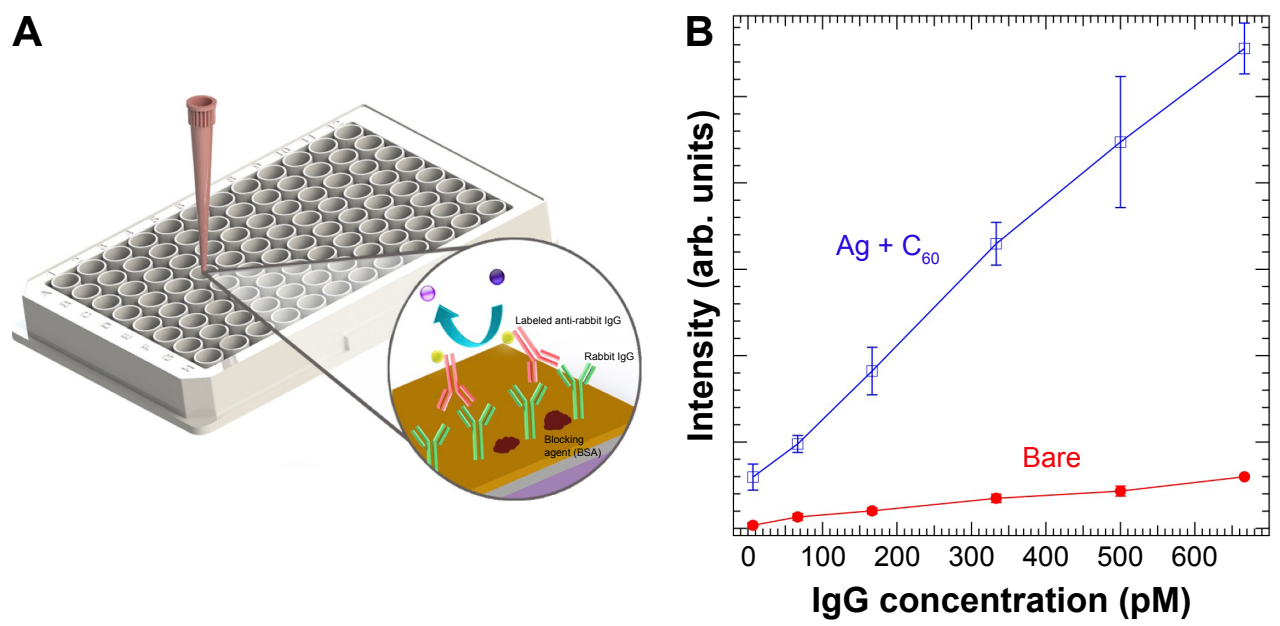

Figure 9 (A) A 96-well plate assay showing the scheme for rabbit $\operatorname{lgG}$ immunoassay. (B) The emission of dye-labeled antirabbit lgG on Ag $+\mathrm{C}_{60}$-coated 96 -well plate showed $\sim 10$ times enhancement relative to the uncoated well plate. The slope of the emission intensity vs analyte concentration for $\mathrm{Ag}+\mathrm{C}_{60}$ well plate was an order of magnitude higher than the uncoated plate, leading to an order of magnitude lower limit of detection.

Figure 2E) leading to a bright red color image (Figure 7B). This reduced quenching is also evident in the emission spectrum of Ag-biotin-SA-RhB complex (Figure 6C), which displayed some weak features above $600 \mathrm{~nm}$. As expected, the increase in the thickness of $\mathrm{C}_{60}$ to $30 \mathrm{~nm}$ decreases the emission relative to $\mathrm{Ag}$ and $\mathrm{Ag} / 20 \mathrm{~nm} \mathrm{C} 60$ due to a rapid decrease in the plasmon-induced field as a function of distance.

The combination of physical and chemical mechanisms synergistically increases the enhancement factor through a new chemiplasmonic paradigm $\sim 20$-folds without the need for any lenses or focusing optics in a simple normal incidence and collection geometry (Figure 1C). Going beyond the simple demonstration of this chemiplasmonic enhancement, we used this unique feature to realize: 1) smartphone-based POC sensing capabilities and 2) sensing in a high-throughput 96-well plate.

As shown in Figure 8A, a smart phone camera was used to capture the emitted light by exciting the substrates with a conventional laser pointer (see Methods section). We did not observe any detectable emission for $\mathrm{RhB}$ on the bare glass, while $25 \mathrm{~nm} \mathrm{Ag}, 20 \mathrm{~nm} \mathrm{C}_{60}$, and $\mathrm{Ag} / 20 \mathrm{~nm} \mathrm{C}_{60}$ exhibited bright emission spots (Figure $8 \mathrm{~B}-\mathrm{I}$ ). The emission from the Ag substrate appeared more yellow than the emission from $\mathrm{C}_{60}$ and $\mathrm{Ag} / \mathrm{C}_{60}$ due to the quenching of red emission, as expected (Figure 2D). More importantly, the emission was clearly the brightest for $\mathrm{Ag} / 20 \mathrm{~nm} \mathrm{C} \mathrm{C}_{60}$. The analysis of red, green, blue or RGB intensity values showed that the counts for $\mathrm{Ag} / 20 \mathrm{~nm} \mathrm{C}{ }_{60}$ were $\sim 17$ times higher, indicating that it is a robust and sensitive platform for POC detection (Figure 8B-I). Another important advantage of using $\mathrm{Ag} / \mathrm{C}_{60}$ films is that the red emission is not completely quenched. The Rayleigh scattering effects, which increase with decreasing wavelength, play a critical role in reducing the emission intensity reaching the smartphone camera in POC settings. In this regard, $\mathrm{Ag} / \mathrm{C}_{60}$ films that support longer wavelength red emission and $\sim 17-20$ times enhancement in a simple geometry without focusing optics are ideal for POC biosensors.

Lastly, in addition to an instrument-free POC setting, a high-throughput 96-well plate assay based on chemiplasmonic enhancements is also demonstrated (Figure 9A). To test the applicability of the presented technology, a model antigen (rabbit IgG) and antibody (labeled antirabbit IgG) were used. On average, the labeled antirabbit IgG showed 10 times enhancement in its emission with $\mathrm{Ag}$ and $\mathrm{C}_{60}$ coatings with an order of magnitude lower limit of detection $\sim 6.6 \mathrm{pM}$ (Figure 9B). These results provide compelling evidence for the utility of chemiplasmonic sensors in practical biochemical applications.

In this article, we showed that a synergistic combination of physical enhancement through Ag surface plasmons and the chemical interactions of dyes with $\mathrm{C}_{60}$ results in a new chemiplasmonic paradigm. While the physical plasmonic mechanism shows six-fold enhancement and the chemical interactions exhibit $\sim 12$-fold enhancement, $\sim 20$-fold enhancement can be achieved in the chemiplasmonic paradigm. We demonstrated two model assays using biotin-SA and rabbit immunoglobulin assay to demonstrate the practical applications of the chemiplasmonic sensors. Our results show clear evidence that chemiplasmonic sensors can be extended to detect biomarkers in a POC setting through a 
smartphone in simple normal incidence geometry without the need for focusing optics. Furthermore, chemiplasmonic sensors also facilitate high-throughput screening of biomarkers in the conventional 96-well plate format with 10 times higher sensitivity.

\section{Acknowledgments}

RP is grateful to the Department of Physics and Astronomy, Clemson University, for providing the start-up funds. The authors also thank Bishwambhar Sengupta, Department of Physics, Clemson University, for his initial help with the data collection.

\section{Author contributions}

All authors contributed to data analysis, drafting or revising the article, gave final approval of the version to be published, and agree to be accountable for all aspects of the work.

\section{Disclosure}

The authors report no conflicts of interest in this work.

\section{References}

1. Vasan AS, Mahadeo DM, Doraiswami R, Huang Y, Pecht M. Point-ofcare biosensor system. Front Biosci. 2013;5(1):39-71.

2. Roda A, Michelini E, Zangheri M, di Fusco M, Calabria D, Simoni P. Smartphone-based biosensors: a critical review and perspectives. $\operatorname{Tr} A C$ Trends Analyt Chem. 2016;79:317-325.

3. Tokel O, Inci F, Demirci U. Advances in plasmonic technologies for point of care applications. Chem Rev. 2014;114(11):5728-5752.

4. Hu J, Wang S, Wang L, et al. Advances in paper-based point-of-care diagnostics. Biosens Bioelectron. 2014;54:585-597.

5. World Health Organization. World Health Statistics 2014. 2014;175. Available from: https://www.who.int/gho/publications/world_health_ statistics/2014/en/. Accessed September 6, 2018.

6. Peeling RW, Mabey D. Point-of-care tests for diagnosing infections in the developing world. Clin Microbiol Infect. 2010;16(8):1062-1069.

7. Choi B, Iwanaga M, Miyazaki HT, Sugimoto Y, Ohtake A, Sakoda K. Overcoming metal-induced fluorescence quenching on plasmo-photonic metasurfaces coated by a self-assembled monolayer. Chem Commun. 2015; 51(57):11470-11473.

8. Mulpur P, Yadavilli S, Mulpur P, et al. Flexible Ag-C60 nano-biosensors based on surface plasmon coupled emission for clinical and forensic applications. Phys Chem Chem Phys. 2015;17(38):25049-25054.

9. Mulpur P, Podila R, Lingam K, et al. Amplification of surface plasmon coupled emission from graphene-Ag hybrid films. J Phys Chem C. 2013; 117(33):17205-17210.
10. Chen C-W, Wang C-H, Wei C-M, et al. Highly sensitive emission sensor based on surface plasmon enhanced energy transfer between gold nanoclusters and silver nanoparticles. J Phys Chem C. 2010; 114(2):799-802.

11. Fu Y, Lakowicz JR. Enhanced fluorescence of Cy5-labeled oligonucleotides near silver island films: a distance effect study using single molecule spectroscopy. J Phys Chem B. 2006;110(45):22557-22562.

12. Lakowicz JR, Malicka J, Gryczynski I, Gryczynski Z. Directional surface plasmon-coupled emission: a new method for high sensitivity detection. Biochem Biophys Res Commun. 2003;307(3):435-439.

13. Enderlein J, Ruckstuhl T. The efficiency of surface-plasmon coupled emission for sensitive fluorescence detection. Opt Express. 2005; 13(22):8855-8865.

14. Gryczynski I, Malicka J, Gryczynski Z, Lakowicz JR. Surface PlasmonCoupled Emission with Gold Films. J Phys Chem B. 2004;108(33): 12568-12574.

15. Lakowicz JR. Radiative decay engineering 3 . Surface plasmon-coupled directional emission. Anal Biochem. 2004;324(2):153-169.

16. Anger P, Bharadwaj P, Novotny L. Enhancement and quenching of single-molecule fluorescence. Phys Rev Lett. 2006;96(11):113002.

17. Novotny L, Hecht B. Principles of Nano-Optics. Cambridge: Cambridge University Press; 2006.

18. Lakowicz JR. Radiative decay engineering: biophysical and biomedical applications. Anal Biochem. 2001;298(1):1-24.

19. Barnes WL. Fluorescence near interfaces: the role of photonic mode density. J Mod Opt. 1998;45(4):661-699.

20. Zhang Y, Aslan K, Previte MJR, Geddes CD. Metal-enhanced fluorescence: surface plasmons can radiate a fluorophore's structured emission. Appl Phys Lett. 2007;90(5):053107.

21. Feng AL, You ML, Tian L, et al. Distance-dependent plasmon-enhanced fluorescence of upconversion nanoparticles using polyelectrolyte multilayers as tunable spacers. Sci Rep. 2015;5:7779.

22. Srinivasan V, Ramamurthy SS. Purcell factor: a tunable metric for plasmon-coupled fluorescence emission enhancements in cermet nanocavities. J Phys Chem C. 2016;120(5):2908-2913.

23. Cao SH, Cai WP, Liu Q, Li YQ. Surface plasmon-coupled emission: what can directional fluorescence bring to the analytical sciences? Annu Rev Anal Chem. 2012;5(1):317-336.

24. Li Y, Qi D, Song P, Ma F. Fullerene-based photoactive layers for heterojunction solar cells: structure, absorption spectra and charge transfer process. Materials. 2014;8(1):42-56.

25. Zhang Z, Yu Y, Wang P. Hierarchical top-porous/bottom-tubular TiO2 nanostructures decorated with Pd nanoparticles for efficient photoelectrocatalytic decomposition of synergistic pollutants. ACS Appl Mater Interfaces. 2012;4(2):990-996.

26. Mulpur P, Podila R, Ramamurthy SS, Kamisetti V, Rao AM. $\mathrm{C}_{60}$ as an active smart spacer material on silver thin film substrates for enhanced surface plasmon coupled emission. Phys Chem Chem Phys. 2015;17(15): 10022-10027.

27. Ray K, Badugu R, Szmacinski H, Lakowicz JR. Several hundred-fold enhanced fluorescence from single fluorophores assembled on silver nanoparticle-dielectric-metal substrate. Chem Commun. 2015;51(81): 15023-15026. 


\section{Supplementary materials}

The supporting information shows the schematic of rhodamine $\mathrm{B}$ electronic structure and polarization measurements of rhodamine $\mathrm{B}$ on $\mathrm{C}_{60}$ and glass.

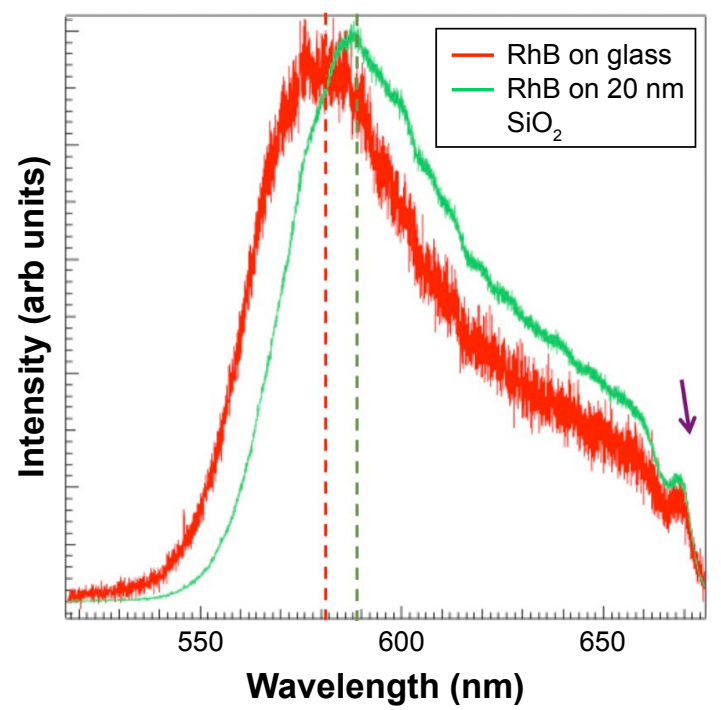

Figure SI The emission spectrum for rhodamine $\mathrm{B}(\mathrm{RhB})$ on bare glass and $20 \mathrm{~nm} \mathrm{SiO}$-coated glass slides. The emission of RhB upshifted (dashed vertical lines) in the presence of $\mathrm{SiO}_{2}$ layer due to the absence of $\pi-\pi$ interactions. The peak $\sim 675 \mathrm{~nm}$ (pointed by the arrow) is used for calibration.
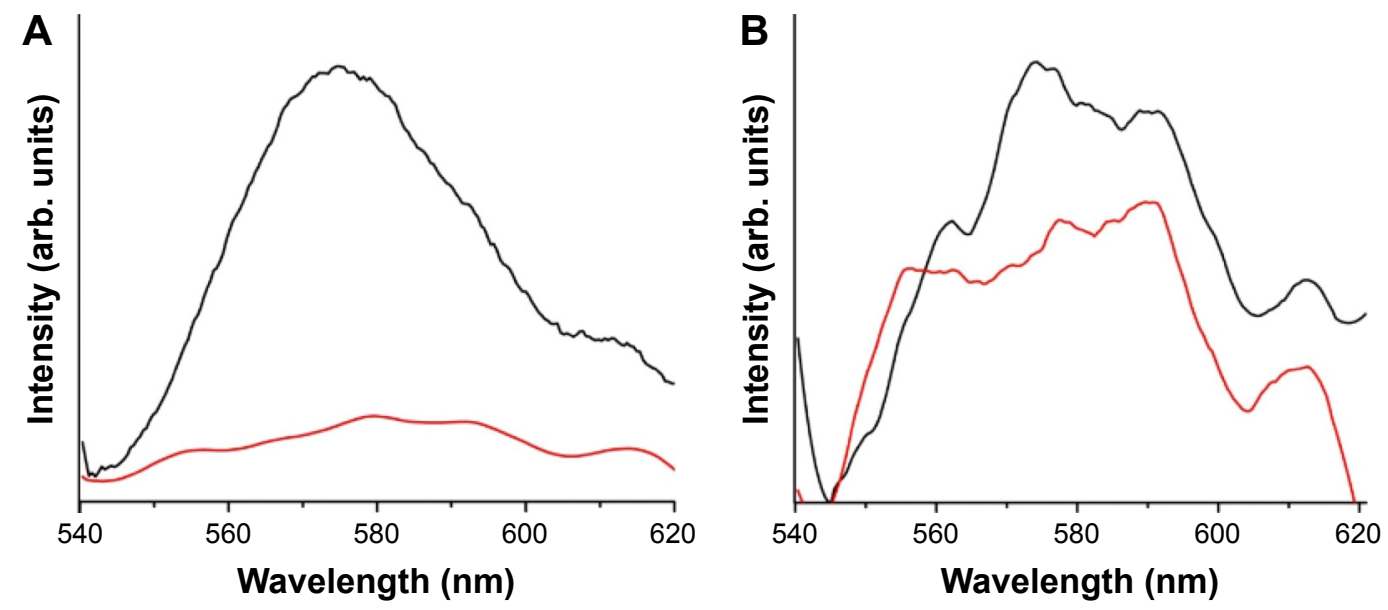

$\mathrm{I}(\mathrm{P})-\mathrm{I}(\mathrm{S})$

Figure S2 Polarized emission intensity plots of rhodamine $B(R h B)$ showing predominantly p-polarized output when coated on glass/ ${ }_{60}$ substrate $(\mathbf{A})$, and the absence of such polarized emission on bare glass (B). The predominant $p$-polarized emission on glass $/ C_{60}$ is attributed to the $\pi-\pi$ interactions between RhB and $C_{60}$. Abbreviations: I(P), p-polarized intensity; I(S), s-polarized intensity. 


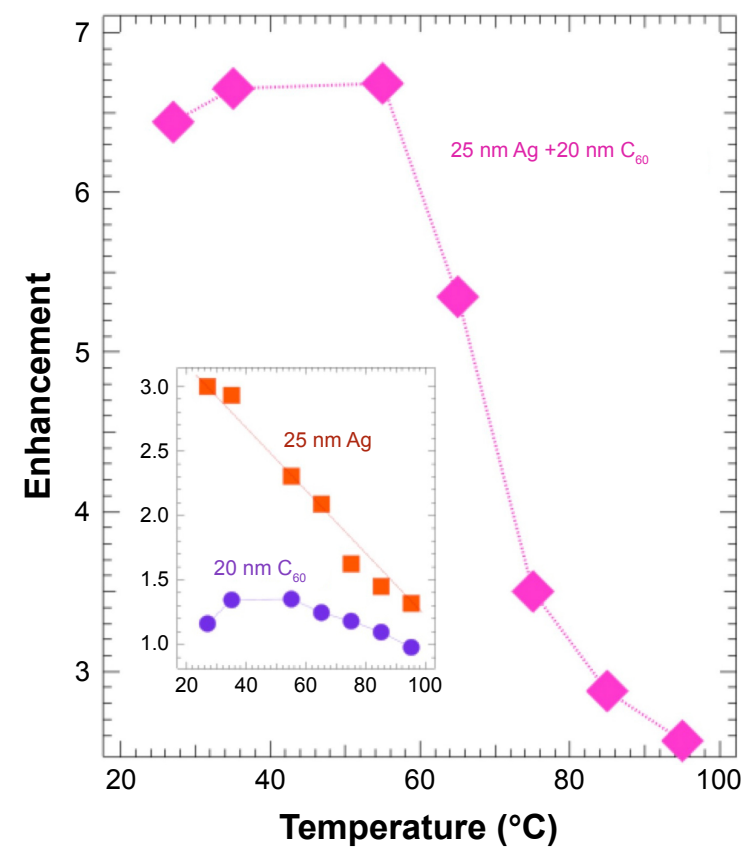

Figure S3 Temperature dependence of the enhancement factor of rhodamine $B(R h B)$ on $20 \mathrm{~nm} C_{60}, 25 \mathrm{~nm} \mathrm{Ag}$, and $25 \mathrm{~nm}$, and $A g+20 \mathrm{~nm} C_{60}$ relative to bare glass substrate.

\section{Publish your work in this journal}

The International Journal of Nanomedicine is an international, peerreviewed journal focusing on the application of nanotechnology in diagnostics, therapeutics, and drug delivery systems throughout the biomedical field. This journal is indexed on PubMed Central, MedLine, CAS, SciSearch ${ }^{\circledR}$, Current Contents ${ }^{\circledR} /$ Clinical Medicine,
Journal Citation Reports/Science Edition, EMBase, Scopus and the Elsevier Bibliographic databases. The manuscript management system is completely online and includes a very quick and fair peer-review system, which is all easy to use. Visit http://www.dovepress.com/ testimonials.php to read real quotes from published authors. 